\title{
Cyclic Reciprocal Sums of Prime Products
}

\author{
Serge Dolgikh [0000-0001-5929-8954] \\ Dept. of Information Technology, \\ National Aviation University
}

\begin{abstract}
We discuss cyclic reciprocal sums of products of primes that appear in distributions of no-prime (NP) sequences. Formulas and asymptotics for cyclic sums of arbitrary order are obtained, and possible connections with certain fields of physics briefly discussed.
\end{abstract}

Keywords: Number theory; prime number distribution; prime sequences

\section{Definitions}

A cyclic reciprocal prime sum of order $K$, and a set of consecutive primes $P: p_{l}<p_{k}$ $<p_{N}$ of length $N$ is defined as the sum of reciprocal unique products of primes in the set:

$$
C_{K}(P, N)=\sum_{p_{1}}^{p_{l}<p_{N}, p_{i} \neq p_{j}} \frac{1}{p_{1} p_{2} \cdot . p_{K}}
$$

Immediately,

$$
C_{1}(P)=\sum_{p_{1}}^{p_{l} \leq p_{N}} \frac{1}{p_{l}}, \quad C_{N}(P)=\frac{1}{p_{1} p_{2} . . p_{N}}
$$

For convenience, in the text that follows the notation $q_{i}=1 / p_{i}$ will be used. We will also denote:

$$
E_{k}(P)=\sum_{p_{1}}^{p_{l} \leq p_{N}} \frac{1}{p_{l}^{k}}, k \geq 1
$$

From the definition, cyclic sums are invariant with respect to the group of transpositions of the set $P$.

\section{Expressions and Asymptotics}

It is well known that $E_{k}, k>1$ has constant asymptotics for $N \rightarrow \infty$ directly related to the prime Riemann function $\xi(k)$ and is unbounded for $k=1$ with the asymptotics of $\log \log p_{N} \sim \log \log (N \log N)[1,2]$.

From the definition, for $N=1, C_{l}(P)=E_{l}(P)$. Then, for $N=2$, straightforwardly: 


$$
\begin{gathered}
E_{1} C_{1}=\sum_{i=1}^{N} q_{i}\left(C_{1}-q_{i}\right)+\sum_{i=1}^{N} q_{i}^{2}=2 C_{2}+E_{2} \\
C_{2}=\frac{1}{2}\left(E_{1}^{2}-E_{2}\right)
\end{gathered}
$$

Same strategy allows to express $C_{k}(P), k=2 \ldots N$ via $E_{1} \ldots E_{k}$ :

$$
E_{1} C_{k}=\sum_{i=1}^{N} q_{i}\left(C_{k}-q_{i} C_{k-1} \backslash q_{i}\right)+\sum_{i=1}^{N} q_{i}^{2} C_{k-1} \backslash q_{i}=(k+1) C_{k+1}+O\left(E_{1}^{k-1}\right)
$$

One can see that $C_{k}(P)$ has the asymptotics:

$$
C_{k}(P)=\frac{1}{k !} E_{1}^{k}+O\left(E_{1}^{k-2}\right)
$$

with the second term,

where $j, l>1$.

$$
O\left(E_{1}^{k-2}\right)=\sum_{i=1}^{k-2} D_{i, j} E_{1}^{i} \Pi E_{j} E_{l}+D_{0} E_{k}
$$

Hence, the behavior of $C_{k}(P)$ is polynomial of the order $k$ with respect to $E_{l}(P)$. As the asymptotics of $\mathrm{E}_{k}$ with $k>1$ are constant, (3) and (4) can be expressed as:

$$
C_{k}(P)=\frac{1}{k !} E_{1}^{k}+\sum_{i=1}^{k-2} D_{i} E_{1}^{i}+D_{0} E_{k}
$$

with $D_{i}=\sum_{i=1}^{k-2} D_{i, j} \Pi E_{j} E_{l}$, the cyclic coefficients. It is easy to see that $D_{0}=\frac{1}{k}$ and so on.

\section{Cyclic Prime Sums and the Naturalness Problem}

Let us consider the decomposition of a cyclic sum by dominant terms (4-6) in the form:

$$
C_{k}(P)=\frac{1}{k !} E_{1}^{k}\left(1+\sum_{i=0}^{k-2} D_{i} E_{1}^{-(k-i)}\right)
$$

Here, $E_{l}(P) \rightarrow \infty$ with $N \rightarrow \infty$ and for each term $T_{i}$ in (7) it should hold: $T_{i+1} / T_{i} \rightarrow 0$ for $N \rightarrow \infty$.

Cyclic prime sums are interesting for their "shaving" effect, whereby the resulting value of the sum can be significantly, many orders of magnitude smaller than the contributing terms.

Consider the examples of cyclic sums of the maximum order $N$ in the Table 1 for the sets of first prime orders with $N=10 ; 15 ; 25, p_{1}=5$. 
Table 1. Cyclic prime sums and term contributions.

\begin{tabular}{cccc}
\hline$N$ & Cyclic sum, $C_{N}$ & First term, $\frac{1}{k !} E_{1}^{k}$ & Last term, $\frac{1}{k} E_{k}$ \\
\hline 10 & $3.35 \times 10^{-13}$ & $1.11 \times 10^{-8}$ & $1.06 \times 10^{-8}$ \\
15 & $8.70 \times 10^{-22}$ & $4.02 \times 10^{-14}$ & $2.20 \times 10^{-12}$ \\
25 & $3.97 \times 10^{-41}$ & $1.36 \times 10^{-26}$ & $1.34 \times 10^{-19}$ \\
\hline
\end{tabular}

With a value of the dominant term for $N=25$ of the order $10^{-26}$, the resulting value of $C_{N}(6)$ had the order of $10^{-41}$. These examples demonstrate that the difference in scale between the resulting value of the sum and the contributing term can reach $10^{-20}$ even for relatively small sets of primes.

Evidently, with higher $N$, the cyclic sum $C_{N}$ is bound to remain positive; and very small, that is, in the order of $1 / p_{N} \# \sim e^{-N} \log N$ [3], regardless of the arithmetics behind the values of the cyclic coefficients $D_{i j}(6)$. These constraints force the relations between $D_{i j}$ and $E_{l}{ }^{k}$ to balance out in a very precise and precarious way to produce extremely small resulting positive values of the higher-order sums, even with the contributing terms of significantly higher magnitude.

Interestingly, this behavior of cyclic sums is reminiscent of some phenomena in High Energy Physics, such as the problem of vacuum contributions of quantum fields and cosmological constant [4-6]. This problem is sometimes commented to have a tension with "the naturalness" assumption, because such a precise balancing of terms can be seen as requiring special assumptions about the contributions. Yet, as seen with the cyclic sums, it can emerge quite naturally in some problems of number theory and prime mathematics.

\section{Cyclic Sums and No-Prime Distributions}

No-prime (NP) sequences were defined [7] as sequences of pairs of arbitrary length with no primes. While a detailed analysis of NP-sequences and distributions will be a subject of another work, conceptually, an approach to understanding distributions of $\mathrm{NP}$-sequences based on prime modulo cycles is relatively straightforward.

Evidently, the necessary and sufficient condition for a sequence of $K$ consecutive non-prime pairs: $\left\{k,\left(x_{1}, y_{1}\right), l_{1},\left(x_{2}, y_{2}\right), \ldots\right\}$ and the key $k$ is a constraint on the prime modulo functions $h_{p}(k)$ to have at least one value in the range $P=\{(1,2),(4,5),(7$, $8), \ldots\}$ representing the sequence of positions with relative distance to the key that cannot be prime, for prime $p<k$ :

$$
\forall v \in P \exists p<k: h_{p}(k)=v
$$

Each combination of values satisfying this condition repeats with a period of $3 \times \Pi p_{k}$, leading to an heuristic estimate of the number of NP-sequences of length $K$ in an interval of length $d$ :

$$
N_{N P}(K, d)=\frac{d K !}{3}\left(C_{2 K}\left(p_{m}\right)-N_{o v}(p)\right)
$$


where $C_{2 K}$ is the cyclic prime sum of the order $2 K, p_{m}$ the maximum prime that can be a factor in the range $d, p_{m} \sim d / 3$ and $N_{o v}$, the count of overlaps between combinations of prime moduli resulting in an NP-condition at certain positions that needs to be subtracted from the sum (9) to prevent counting the positions of overlaps multiple times.

It follows then that distributions of NP-sequences are directly related to cyclic prime sums of even orders.

\section{Conclusion}

In this work a definition and an analysis of cyclic sums of reciprocal products of primes were given. Cyclic reciprocal sums of prime products emerge in some areas of prime number theory such as distributions of no-prime (NP) sequences and have interesting properties, such as "unnaturally" small resulting value of the sum relative to the order of contributing terms. It is possible for these reasons that cyclic reciprocal sums of prime products will find further applications in the fields and problems of mathematics and theoretical physics.

\section{References}

1. Ingham, A.E. The distribution of prime numbers. Cambridge University Press (1990) 2-5.

2. Mertens, F. Ein Beitrag zur analytischen Zahlentheorie. J. reine angew. Math.78(1874) 4662 .

3. Weisstein, E.W. Chebyshev functions. MathWorld, https://mathworld.wolfram.com/ChebyshevFunctions.html.

4. 't Hooft, G. Naturalness, chiral symmetry and spontaneous chiral symmetry breaking". In 't Hooft, G. (ed.). Recent Developments in Gauge Theories. Plenum Press (1980).

5. Burton, R. Is "naturalness" unnatural? In: 14th International Conference on Supersymmetry and the Unification of Fundamental Interactions (SUSY06), (2006).

6. Dolgikh, S. and Schwarz A.S. Supergrassmannians, super $\tau$-functions and strings. Physics and Mathematics of Strings: Memorial Volume for Vadim Knizhnik, (1990) 231-244.

7. Dolgikh, S. Prime sequences and distributions. Cambridge Open Engage. doi:10.33774/coe-2020-g9d1m (2020). 